\title{
Effects of Experemental Cerebral Ishemia on Metabolic Characteristics of Parietal Cortex Neurons
}

\author{
Lizaveta Igorevna Bon ${ }^{1}$, Nataliya Yevgenyevna Maksimovich ${ }^{1}$, Sergey Michailovich Zimatkin ${ }^{2}$ \\ ${ }^{1}$ Department of Pathological Physiology of the Name of D. A. Maslakov, Grodno State Medical University, Grodno, Belarus \\ ${ }^{2}$ Department of Histology, Cytology and Embryology, Grodno State Medical University, Grodno, Belarus
}

Email address:

e_bon@list.ru(L.I. Bon)

\section{To cite this article:}

Lizaveta Igorevna Bon, Nataliya Yevgenyevna Maksimovich, Sergey Michailovich Zimatkin. Effects of Experemental Cerebral Ishemia on Metabolic Characteristics of Parietal Cortex Neurons. Bioprocess Engineering. Vol. 2, No. 1, 2018, pp. 1-5. doi: 10.11648/j.be.20180201.11

Received: February 21, 2018; Accepted: March 9, 2018; Published: April 3, 2018

\begin{abstract}
The aim of the paper was to estimate the effect of experimental cerebral ischemia on metabolic characteristics of parietal cortex neurons. The experiments were carried out on 12 laboratory male rats. Cerebral ischemia was modeled with bilateral joint carotid arteries with durations of 60 minutes under intravenous thiopental anesthesia $(40-50 \mathrm{mg} / \mathrm{kg})$. The rats were decapitated and the samples of the brain parietal cortex were prepared for histological and histochemical examination in combination with morphometry to examine the $5^{\text {th }}$ layer inner pyramidal neurons. It was increased the number of pathological forms and shrinkage of the $5^{\text {th }}$ layer inner pyramidal neurons after cerebral ischemia in rats. The histochemical examination was revealed the inhibition of NADH-, glucose-6-phosphate dehydrogenase and succinate dehydrogenases as well as activation of lactate dehydrogenase and acid phosphatase. Cerebral ischemia induces deep histological and histochemical changes in the parietal cortex neurons in rats, including shrinkage of parietal cortex neurons and disturbances of their energy metabolism.
\end{abstract}

Keywords: Cerebral Ischemia, Parietal Cortex, Neurons

\section{Introduction}

Cerebral ischemia is a severe neurodegenerative disorder that, depending on the area affected, could impede a variety of physiological functions, including cognitive and motor disturbances. According to the published data, the cerebral cortex is particularly sensitive to ischemia. Cerebral ischemia induces apoptosis, degeneration, reduction the size of brain cortex neurons, the decrease in their protein content, hypoplasia of cytoplasm, significant ultrastructural abnormalities in them. Cerebral ischemia induces defects in many molecular, neurochemical and cellular processes that occur during normal brain functions, regulation cell-cell interactions, increases the formation of free radicals, decreases the content of neurotrophic factors in the brain. Such changes may underlie some of CNS abnormalities related to the cerebral ischemia $[1,8,12]$.

The aim of the present study is to estimate the effect of incomplete ischemia on histological and histochemical characteristics of parietal cortex neurons in rats.

\section{Materials and Methods}

\section{Animals, chemicals and experimental design}

12 male rats were obtained from the breeding colony of the Grodno State Medical University. Their weight was $230 \pm 29$ g. All experimental procedures complied with European Community Council Directive (86/609/EEC) for care and use of laboratory animals. Protocols were reviewed and approved by the Ethical Committee of the Grodno State Medical University (protocol No 1, 14.04.2013). All efforts were made to minimize animal suffering. Rats were housed in vivarium with free access to standard laboratory food and kept under controlled environmental conditions. Incomplete ischemia was modeled by bilateral superimposing ligatures into common carotid arteries the durations of 60 minutes under intravenous thiopental anesthesia $(40-50 \mathrm{mg} / \mathrm{kg})$. The rats were decapitated and the samples of the brain parietal cortex were prepared for histological and histochemical examination in combination with morphometry to examine the $5^{\text {th }}$ layer inner pyramidal neurons.

All the chemicals were obtained from Sigma-Aldrich 
(USA).

\section{Histology}

6 controls and 6 rats after incomplete ischemia were decapitated after 60 minutes and their brain was removed. Samples of the parietal brain cortex were fixed in the mixture of alcohol, chloroform and acetic acid in the ratio 6:3:1, then treated with alcohol and xilen and embedded in paraffin. The $7 \mu \mathrm{m}$ sagittal sections of the parietal cortex were prepared using microtome (Leica RM2125, Germany). They were stained with $0.1 \%$ solution of thionine (the Nissl method) to assess general cytology of neurons.

Histochemistry

Pieces of parietal cortex were then obtained, frozen and stored in liquid nitrogen for further analysis. The $10 \mu \mathrm{m}$ serial sagittal sections of the frozen frontal cortex were prepared using cryostat (Leica CM 1840, Germany). The activity of the oxidative enzymes, such as succinate dehydrogenase (SDH, EC 1.3.99.1), lactate dehydrogenase (LDH, EC 1.1.1.27), glucose-6-phosphate dehydrogenase (G6-PDH, EC 1.1.1.49) and NADH dehydrogenase (NADHDH, EC, 1.1.1.49), as well as the activity of marker lysosomal enzyme acid phosphatase (AP, EC 1.4.3.4) were examined [7]. For the enzyme histochemistry the cryostat sections were placed into the corresponding incubation medium, including the buffer, substrate, co-factor, if necessary, and chromogen to visualize the location of enzymatic activity, for $30 \mathrm{~min}-5$ hours, then washed and embedded in the suitable plastic medium.

Light microscopy and morphometry

For the identification of parietal cortex in the brain sections the stereotaxic atlas was used [6]. The examination of histological preparations, their microphotography and morphometry was carried out using microscope Axioskop 2 plus (Zeiss, Germany) equipped with digital camera (Leica DFC 320, Germany) and computer image analysis software Image Warp (Bit Flow, USA). In preparations stained by the Nissl method all visible neurons of the $5^{\text {th }}$ layer were estimated according to their type of chromatophilia (the intensity of staining of neurons cytoplasm) and divided into normochromic (normal, medium staining), hyperchromic (intense staining), hyperchromic shrinkage, hypochromic (pale staining) and cell-shadows (very pale remnants of dead neurons). Three sections of the parietal cortex brain for three fields of vision for each slice were taken. Ten neurons of the $5^{\text {th }}$ layer of the parietal cortex were measured in every field of vision.

To estimate the size and shape of neuronal bodies the images of up to 30 neurons bodies on the computer monitor were outlined by mouse cursor. Area, form-factor $\left(4 \pi \mathrm{S} / \mathrm{P}^{2}-\right.$ parameter of sphericity and folding) and factor of elongation (maximal D/minimal D - parameter of sphericity) were calculated.

The enzyme activities were determined in cytoplasm of neurons on the optic density of chromogen obtained in the course of histochemical reactions.

\section{Statistics}

The mean values obtained for every animal were processed with nonparametric statistics (because of the small number of animals in the groups) using software STATISTICA 10.0 (StatSoft, Inc., USA). In descriptive statistics, the values of median $(\mathrm{Me})$ and interquartile range (IQR) were determined. The differences were considered significant at $p<0.05$ (Mann-Whitney U-test), because it was not a normal distribution.

\section{Results}

Histology

After incomplete ischemia the area and form-factor of the neuron bodies was significantly lower, but factor of elongation increased, as compared to controls (Table 1).

Table 1. The size and shape of perikaryon of the $5^{\text {th }}$ layer neurons in the parietal cortex of rats. Data are presented as $\mathrm{Me}(L Q ; U Q)$.

\begin{tabular}{ll}
\hline Area, $\mathbf{~ m c m}^{2}$ & \\
\hline control & $170(110 ; 204)$ \\
incomplete ischemia & $72.5(68 ; 75)^{*}$ \\
form-factor & \\
$\begin{array}{l}\text { control } \\
\text { incomplete ischemia }\end{array}$ & $0.92(0.9 ; 0.94)$ \\
factor of elongation & $0.8(0.75 ; 0.85)^{*}$ \\
control & $1.3(1.25 ; 1.35)$ \\
incomplete ischemia & $1.5(1.4 ; 1.6)^{*}$ \\
\hline
\end{tabular}

* $-\mathrm{p}<0.05$, as compared to controls.
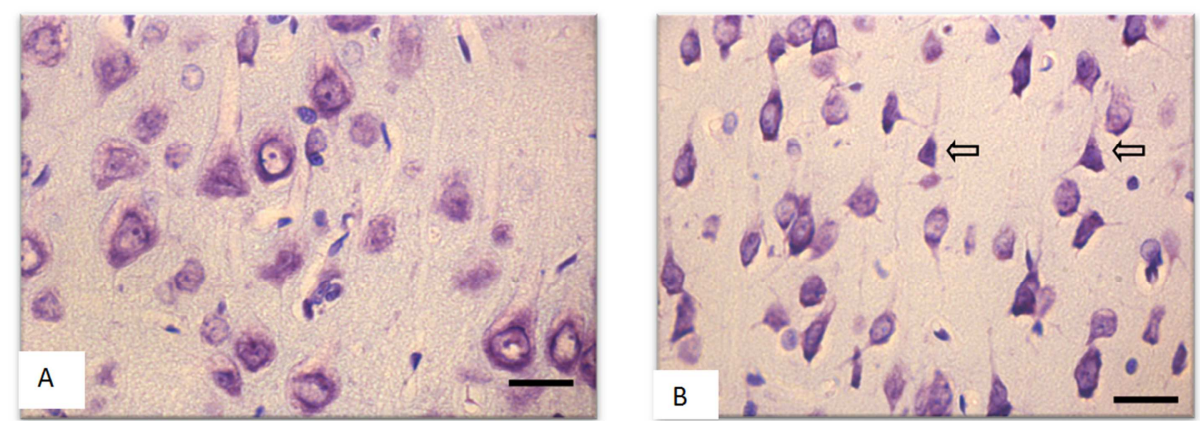

Figure 1. The $5^{\text {th }}$ layer parietal cortex neurons on the $90^{\text {th }}$ postnatal day in controls (A) and rats after incomplete ischemia (B). The arrow shows the shrinkage of hyperchromatic neurons. Stained by the Nissl method. Digital microphotography. Scale bars- 20 um, magnifications- 40x.

In control animals normochromic neurons in preparations of parietal brain cortex prevailed (60-70\%) (Figure 1 A, 2).
After the incomplete ischemia the number of normochromic neurons decreased significantly and the 
number of abnormal neurons (hyper-, hypochromic neurons and cell-shadows) increased (Figure $1 \mathrm{~B}, 2$ ). The amount of shrinking hyperchromic and cell-shadows neurons in rats after subtotal ischemia dramatically increased. It is associated with the changes in neurons shape: increase in their elongation and decrease in form factor (sphericity).

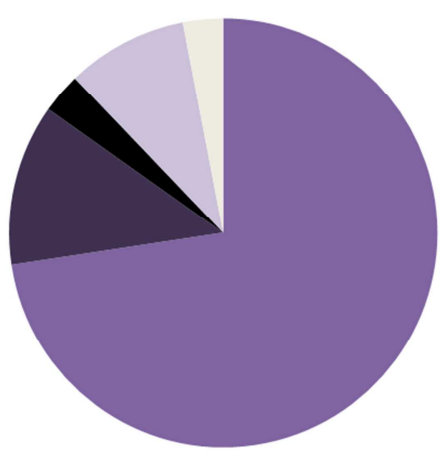

control

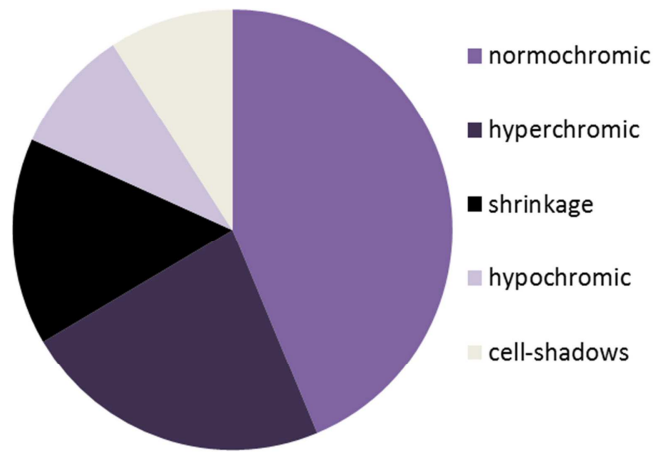

incomplete ischemia

Figure 2. The percentage of neurons with different chromatophilia of cytoplasm in the parietal cortex, \%.

\section{Histochemistry}

Histochemical investigation of parietal brain cortex of rats following incomplete ischemia demonstrated the inhibition of NADH-, glucose-6-phosphate and succinate dehydrogenases and activation of lactate dehydrogenase and acid phosphatase in cytoplasm of $5^{\text {th }}$ layer pyramidal neurons (Figure 3, 4, Table 2).
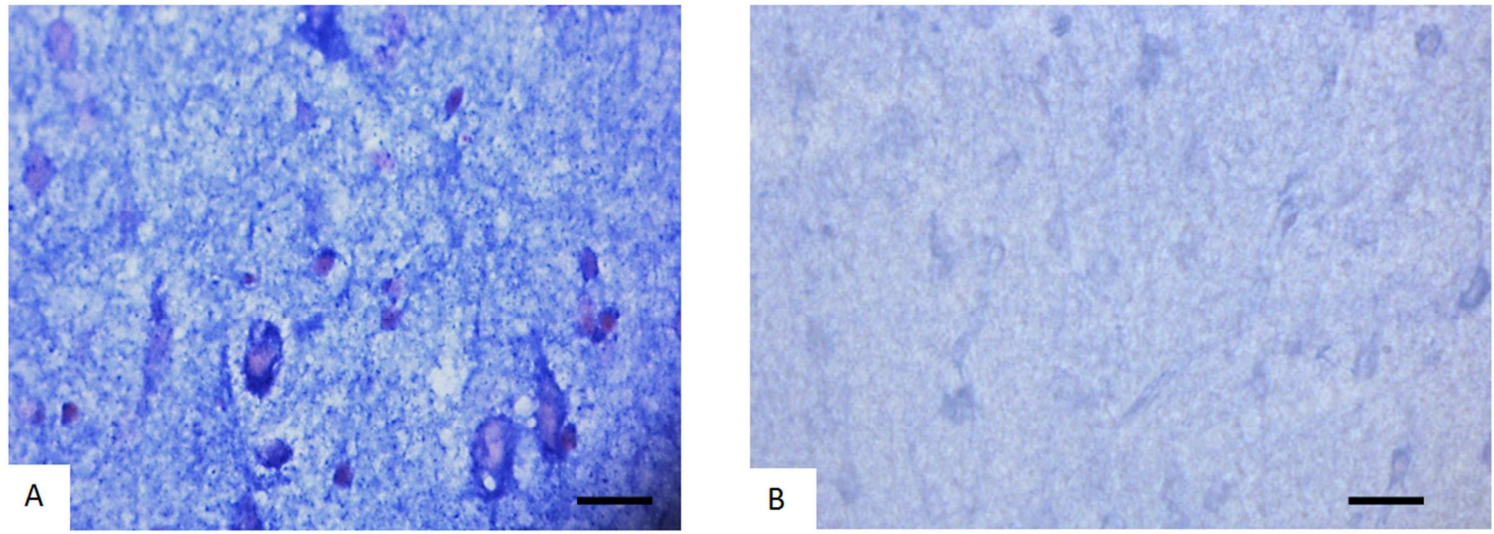

Figure 3. Activity of NADH-dehydrogenase in the $5^{\text {th }}$ layer parietal cortex neurons in control (A) and rats after incomplete ischemia (B). Digital microphotography. Scale bars - $20 \mu \mathrm{m}$, magnifications $-40 x$.
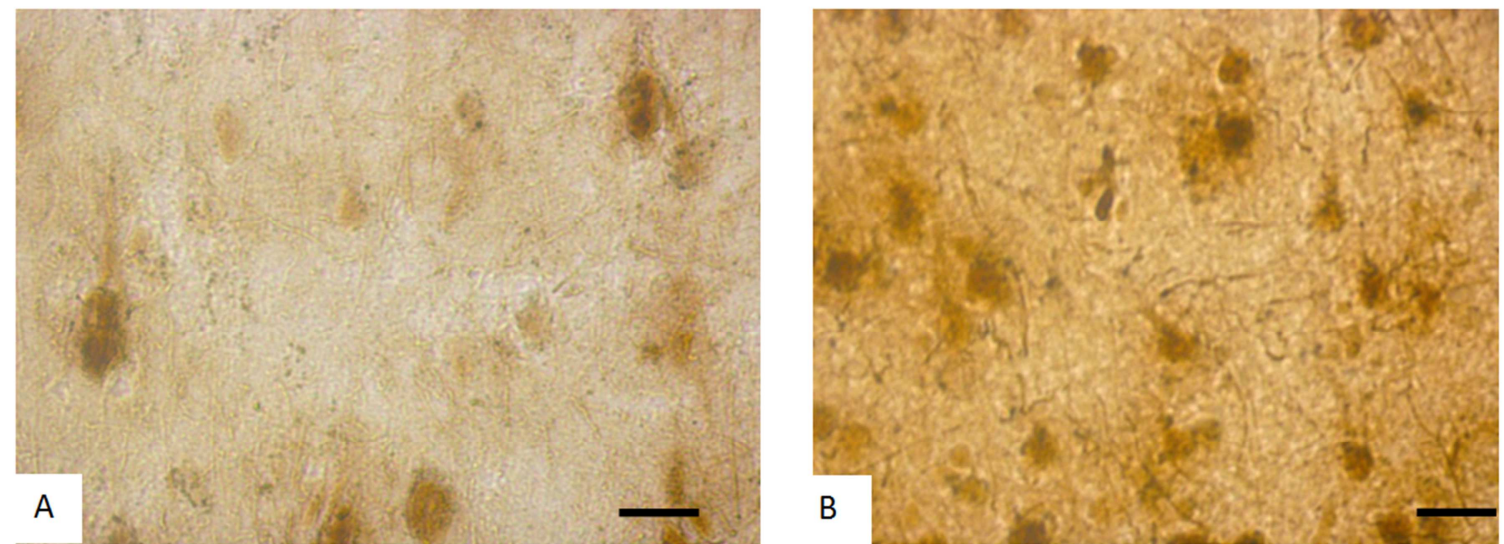

Figure 4. Activity of acid phosphatase in the $5^{\text {th }}$ layer parietal cortex neurons in control (A) and rats after incomplete ischemia (B). Digital microphotography. Scale bars $-20 \mu \mathrm{m}$, magnifications $-40 x$. 
Table 2. The activity of the oxidative enzymes of the $5^{\text {th }}$ layer neurons in the parietal cortex of rats. Data are presented as $\operatorname{Me}(L Q ; U Q)$.

\begin{tabular}{ll}
\hline NADH-dehydrogenases & $0.21(0.20 ; 0.26)$ \\
\hline $\begin{array}{l}\text { control } \\
\text { incomplete ischemia }\end{array}$ & $0.16(0.15 ; 0.18)^{*}$ \\
$\begin{array}{l}\text { Succinate dehydrogenase } \\
\text { control }\end{array}$ & $0.18(0.16 ; 0.19)$ \\
$\begin{array}{l}\text { incomplete ischemia } \\
\text { Glucose-6-phosphate }\end{array}$ & $0.11(0.1 ; 0.12)^{*}$ \\
control & $0.23(0.22 ; 0.25)$ \\
incomplete ischemia & $0.16(0.13 ; 0.19)^{*}$ \\
$\begin{array}{l}\text { Lactate dehydrogenase } \\
\text { control }\end{array}$ & $0.11(0.1 ; 0.13)$ \\
$\begin{array}{l}\text { incomplete ischemia } \\
\text { Acid phosphatase } \\
\text { control } \\
\text { incomplete ischemia }\end{array}$ & $0.14(0.13 ; 0.15)^{*}$ \\
\hline
\end{tabular}

$*_{-} \mathrm{p}<0.05$, as compared to controls.

\section{Discussion}

After the ischemia we found the increase in the amount of abnormal forms of neurons. Decrease in the number of normochromic neurons and increase in the number of pathological forms of neurons (hyper-, hypochromic neurons, shrinking hyperchromic neurons and cells shadows) in the 5 th layer of the parietal cortex was found. Hyperchromic neurons usually had much more free ribosomes, which ensures their hyperchromy by the Nissl method staining. This testifies the intensive biosynthesis of proteins for the own needs of neurons. Perhaps this is a way of adaptation of brain cortex neurons to compensate the hypoxia. Probably a shrinkage of the hyperchromic neurons may be seen as a failure of adaptation that leads to their subsequent death [4].

The activity of the marker aerobic oxidative enzymes (SDH, NADHDH and NADPhHDH) in brain cortex neurons cytoplasm decreased, but the marker enzyme of anaerobic glycolysis, lactate dehydrogenase increased. It indicates probably both the disturbances of energy metabolism and metabolic adaptation of neurons to cerebral ischemia [1-3].

As it is known, on the ultrastructural level, a brain cortex neurons after incomplete ischemia displayed degenerative changes. The destructive changes in mitochondria (some of them were swelling, and showing the decrease in the density of cristae) corresponding to the decrease in activity of their marker enzymes, succinate and $\mathrm{NADH}$-dehydrogenases. A reduction of bind ribosome number and RER canals indicate a decreased of protein biosynthesis for export to nerve terminals. The significant increase in the amount and size of lysosomes in neurons accompanied by an activation of the lysosomal marker enzyme acid phosphatase may reflect the increased autophagy for the removal of damaged microstructures $[1,3$, 4].

Mechanisms of cell damage under ischemia include excitotoxicity, oxidative stress, free radical production, apoptosis and inflammation. Oxidative stress leading to calcium accumulation, mitochondrial dysfunction and the production of reactive oxygen radicals is an important mechanism of cell death following ischemia. It leads to potassium-induced release of excitatory amino acids, the opening of glutamate receptor associated ion channels and the influx of $\mathrm{Ca}^{2+}$ [3]. The role of nitric oxide (NO) in the pathophysiology of cerebral ischemia has also been documented. In ischemic models, abnormal NO production may be both beneficial and detrimental, depending upon when and where this neurotransmitter is released. Increased inducible NO form of activity generally occurs in a delayed fashion after brain ischemia and is associated with inflammatory processes $[8,9,10,11,12]$.

\section{Conclusion}

Identified morphological and metabolic disturbances in the parietal cortex neurons may underlie the known neurological and behavioral disorders in animals and human after cerebral ischemia [5]. In conclusion, cerebral ischemia in rats induces deep structural and metabolic disturbances in the parietal cortex neurons.

\section{Funding}

Grodno State Medical University grant for reagents and animals.

\section{Conflict of Interest}

None declared.

\section{References}

[1] Borges N., Cerejo A., Santos A. (2004) Changes in rat cerebral mitochondrial succinate dehydrogenase activity after brain trauma. Int. J. Neurosci., 114, 217-227.

[2] Chalmers G. R., Roy R. R., Edgerton V. R. (1992) Adaptability of the oxidative capacity of motoneurons. Brain. Res., 570, 1-10.

[3] Chan P. H. (2004) Mitochondria and neuronal death/survival signaling pathways in cerebral ischemia. Neurochem. Res., 29, 1943-1949.

[4] Gallyas F (2007) Novel cell-biological ideas deducible from morphological observations on "dark" neurons revisited. Ideggyogy. Sz. 78, 212-222.

[5] Hamilton G. F. (2016) Behavioral deficits induced by thirdtrimester equivalent alcohol exposure in male C57BL/6J mice are not associated with reduced adult hippocampal neurogenesis but are still rescued with voluntary exercise. Behav Brain Res., 45, 615-620.

[6] Paxinos G \& Watson C (2007) The rat brain in stereotaxic coordinates (6th ed). London: Academic Press.

[7] Pearse, A. G. E. (1960). Histochemistry: theoretical and applied (2nd ed). London: Churchill. 
[8] Stagliano, NE, Dietrich, WD, Prado, R, Green, EJ, Busto, R (1997) The role of nitric oxide in the pathophysiology of thromboembolic stroke in the rat. Brain Res 759:32-40

[9] Wada, K, Chatzipanteli, K, Busto, R, Dietrich, WD (1998a) The role of nitric oxide in traumatic brain injury in the rat. $\mathrm{J}$ Neurosurgery 89:807-818.

[10] Zauner, A, Bullock, R, Kuta, AJ, Woodward, J, Young, HF (1996) Glutamate release and cerebral blood flow after severe human head injury. Acta Neurochir Suppl 6740:6744.
[11] Zhang, F, Sprague, SM, Farrokhi, F, Henry, MN, Son, MG, Vollmer, DG (2002) Reversal of attenuation of cerebrovascular reactivity to hypercapnia by a nitric oxide donor after controlled cortical impact in a rat model of traumatic brain injury. J Neurosurg 97:963-969.

[12] White B. C. (2000) Brain ischemia and reperfusion: molecular mechanisms of neuronal injury. J. Neurol. Sci., 176, 1-33. 